\title{
INTELLIGENT COLOR BASED OBJECT SORTING USING ROBOTICS
}

\section{RANI A. MAGADUM \& DR. UTTAM L. BOMBALE}

Electronics Technology, Department of Technology, Shivaji University, Kolhapur, Maharashtra India

\begin{abstract}
The color-based object sorting is required in a variety of industries such as fruit, vegetable sorting, and packaging industry. The processing is complex in human controlled environments and hence accuracy and efficiency decrease with time for a large volume of objects to be sorted. This triggers the requirement of automation implementation to keep accuracy and efficiency for the long term and large volume processing. When color based object sorting is atomized, in the existing system, the only color of an object is recognized and hence when collecting buckets locations are reshuffled, the problem of misplacing of an object takes place. In this paper, we have addressed few of the existing color based sorting systems and have shown the implementation of color based object sorting with the addition of sensor-based intelligence for color recognition of collecting buckets for solving bucket reshuffling problem. The object color recognition is done by image processing and bucket color is recognized with the help of color recognition sensors which shows reliability and accuracy in processing. The reshuffling recognition is the novelty of our implemented work shown in this paper.
\end{abstract}

KEYWORDS: Robotic Arm, Color Recognition, Collecting Bucket Reshuffling \& Object Sorting

Received: Mar 19, 2019; Accepted: Apr 09, 2019; Published: May 06, 2019; Paper Id.: IJRRDJUN20195

\section{INTRODUCTION}

Improvement of processing speed, reduce labor requirements along with better accuracy and efficiency are the main requirements of modern industry.

In the field of science and technology, Machine Vision (MV) is a young discipline. For over 35 years field of MV has been constantly evolved and has become a useful tool in industrial processes. (Davies 2012). Counting, quality control, measurement object sorting and etc. are applications of MV based processing. It ultimately reduces the requirement of labor and increases efficiency along with speed of processing and becomes most promising profitable and long term solution scenario for industries.

Extracting important information of image scenes is the main processing approach in Digital Image processing. (Wise geek, 2011). Digital Images are combinations of different light intensity levels. The pixel which is a point in the image informs corresponding light intensity along with color information at that point. Different properties and features of an image can be extracted in digital image processing with the application of MV. The features may include object shapes and sizes along with color, texture. These feature extractions require complex processing strategies and hence such algorithms are run on the computer with the help of image processing platforms.

The color-based feature extraction can be used for the sorting of objects having different colors when are in a mixed state. The color information extraction can be done RGB color space or also in HSV color space. Most of the time, on the basis of a variety of literature it can be concluded that, HSV color space is 
mostly useful while focusing on actual and accurate color information extraction. In HSV color space hue value related with a particular pixel is nothing but histogram of particular color associated with that pixel. [1]

The normal image is in RGB color space which is required to be converted into HSV color space while extracting hue information. This process requires very less computations and hence easy to implement. The color-based information has a variety of applications in industry. The food industry is one of the main applications where human based sorting for a large volume of items requires a long time and hence robotic based approach along with MV can provide a better solution. Also, this type of implementation can never compromise with the accuracy as human-based approach degrades the efficiency and hence increases mistakes thereby decreasing accuracy as time passes for long time processing scenarios. [2]

In this paper, we have shown the development of color based object sorting robotic arm which involves image processing based color recognition along with a novel approach of sensor-based color recognition of collecting buckets.

\section{RELATED WORK}

There are varieties of techniques available for object sorting developed so far by various researchers out of which we are addressing few here for forming the platform concept of our system.

Amitesh Sachdeval [3] have shown a conveyor belt approach for object sorting purpose applicable for industrial scenarios. The separator based objects are separated by means of machine vision based color recognition. Also for avoiding misprocessing due to limited processing ability of image processing based approach used by authors, whenever required human based inspection requirement is indicated with indicator. The limitations seen for such indicator requirement is overlapping regions of the object while processing the images taken by the camera and having limited scope for providing a solution in terms of physical location rearrangement.

The information on the link on Wikipedia [4], presents sorting of objects based their color in continuous flow based system. The USB interfaced camera is used to process images taken on the specific location of the conveyor. The MATLAB based image processing approach is used to identify color and sort in a respective manner with robotic arm arrangement on the conveyor belt. The microcontroller ATMEGA 328 based electronic system is used for robotic arm for further actions. Pick and place-based processing is done for sort objects based their color and collecting in the respective bucket.

Trinesh, T. M. and Vijayavithal Bongale [5] have shown another way of sorting; in which the color of an object is recognized by using a color sensor. The robotic arm based object pick and place is done to sort objects based on their color.

N. R. Vange et al [6] have color based object sorting with the use of robotic arm and wireless camera. The camera is interfaced with PC for image processing and via serial RS232 communication robotic arm movement is controlled to sort the objects.

U Amin et al [7] have shown the development of robotic arm. The object is picked and placed at the desired location based on its color recognized. TCS 3200 color sensor for object color recognition and Light Dependent Resistor (LDR) is used at the locations to sense respective location feedback based on which object is placed at that 
respective location.

Dharmannagari, Vinay Kumar Reddy [8] have developed a robotic arm for handling different types of materials along with their color recognition.

S. Wanve et al [9] have shown color based object sorting for small sized objects. Camera-based image is taken for processing in MATLAB and color is recognized based on which respective signal is sent to the microcontroller via serial communication for performing respective pick and place operation of the robotic arm.

Vishnu R. Kale [10] have development conveyor belt and separator based system for sorting fruits and vegetables. Camera-based image processing is done to recognize the color and quality of fruits and vegetables.

\section{PROPOSED SYSTEM}

The object sorting based on color is the main aim of the proposed system in which the following objectives are considered.

- To develop a robotic arm for pick and place operations as per command received.

- To develop Image processing based color recognition algorithm.

- To develop a color sensor based bucket color identification to handle bucket place reshuffling cases.

The conceptual block diagram of the proposed system is as is shown in figure 1 for the proposed system.

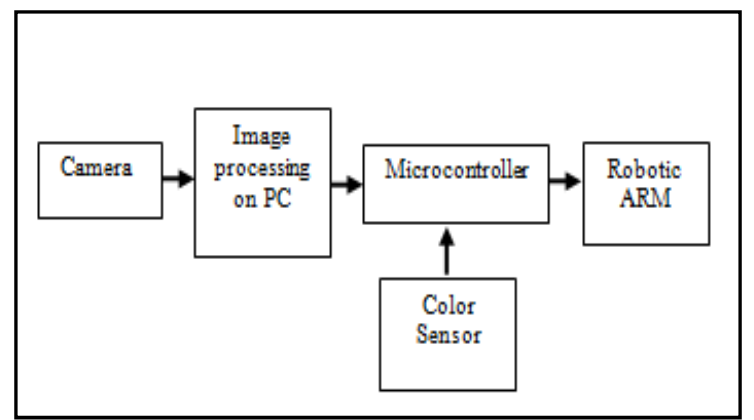

Figure 1: Conceptual Block Diagram of the Proposed System

The system consists of Camera-based image processing for color recognition. The color recognition such as red, green and blue, is achieved by means of converting image from RGB color space to HSV color space. The hue values are evaluated to identify each color. The HSV color model is shown in figure 2. [11, 12]

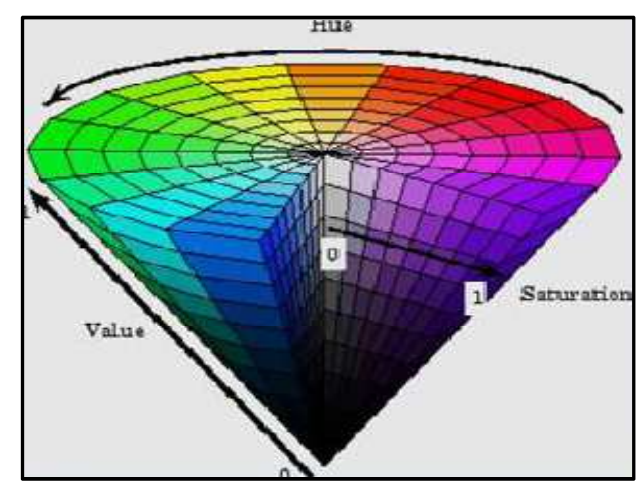

Figure 2: HSV Color Model 
As colors are complexly distributed in color spaces RGB color space model cannot achieve a good color differentiating distance. We use and divide the HSV color space into three subspaces which less complex. The hue distance is modeled based on visual recognition with trial and experimentation method. Also, models with the gray distance and the dark distance are also studied to identify the nature and difference in subspaces. The Euclidian distance-based approach is also comparatively studied in RGB color space and finally use of HSV color space is finalized along with threshold-based approach for better accurate results and based on experimentation and trials.

After various trial and experimentation, it was observed that there is need to have a uniform background. The uniform background is required to achieve better illumination of the object. A black background is used to address this issue. This results in more accurate color recognition of respective objects.

The respective recognized color is assigned specific code which is to be sent to the microcontroller for further processing. The microcontroller receives the respective code and compares with the existing. When respective received code matches with red, green or blue color codes, the respective sort is finalized and movement of the arm is controlled.

When sorting is to be done, objects are to move from their places to drop in the respective bucket and for this, we have developed robotic arm. The arm consists of 4-DOF (Degree of Freedom) movement capability along with the use of four servo motors. The servo motor based approach can provide finalizing respective bucket locations while dropping the object in the respective color bucket. Figure 3 shows the robotic arm developed in stainless steel structure for better rigidity and reliability in a fixed location and positioning operations.

The arm movement with 4-DOF capability gives maximum flexibility while locating buckets for collecting the objects. Also respective desired to bend and to pick operations are also easily achievable through 4-DOF movement. As prototype development, we have limited our approach for 4-DOF but as per requirement in practical applications DOF can also be increased and are easily attachable to existing developed ARM.

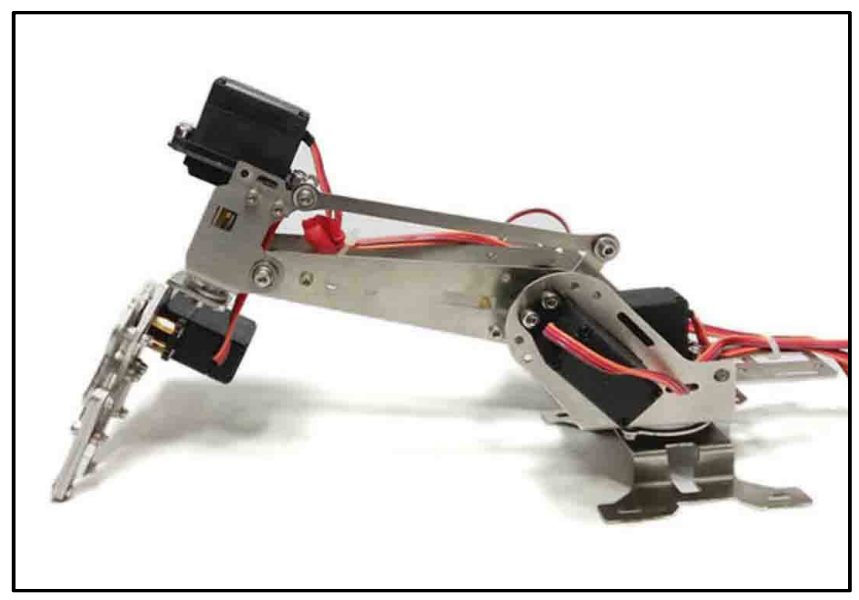

Figure 3: Robotic ARM

When an object is to be dropped in the respective color bucket, the case of reshuffling of the bucket is considered. It may happen that, during a human inspection and monitoring scenarios, the place of the respective color bucket may get changed. To avoid the misplacing of an object, color sensor based bucket color recognition is done. TCS3200 color sensor is placed under each bucket to identify the color of the respective bucket. When the color of 
object matches with that of the bucket then the only object will be dropped in the respective bucket. The placement of the color sensor is shown in figure 4. The operation of the color sensor may get affected due to light illumination entering from the surrounding environment. To avoid entry of the light from sides of the color sensor, which degrades the performance of the sensor, it is covered with black light lockers. The color sensor is equipped with white light LEDs to illuminate the object of which color is to be recognized.

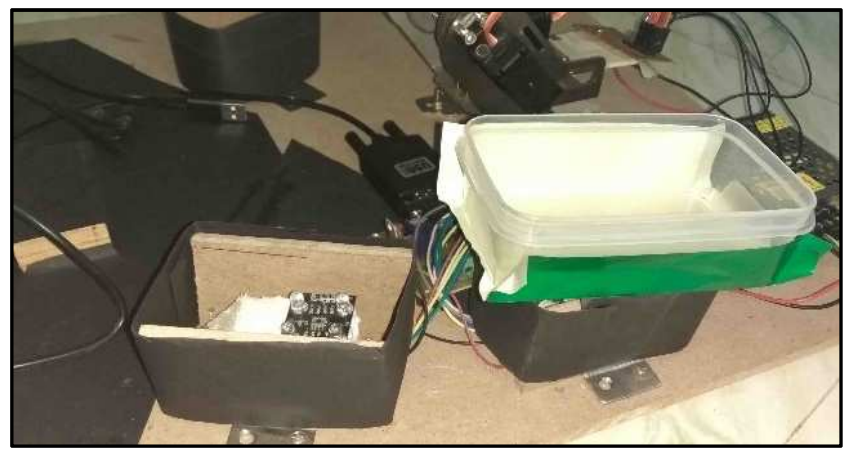

Figure 4: Color Sensor Arrangement for Bucket Color Recognition

The implementation of the robotic arm and sorting arrangement is shown in figure 5. The camera is located well above where the object is to be placed for sorting.

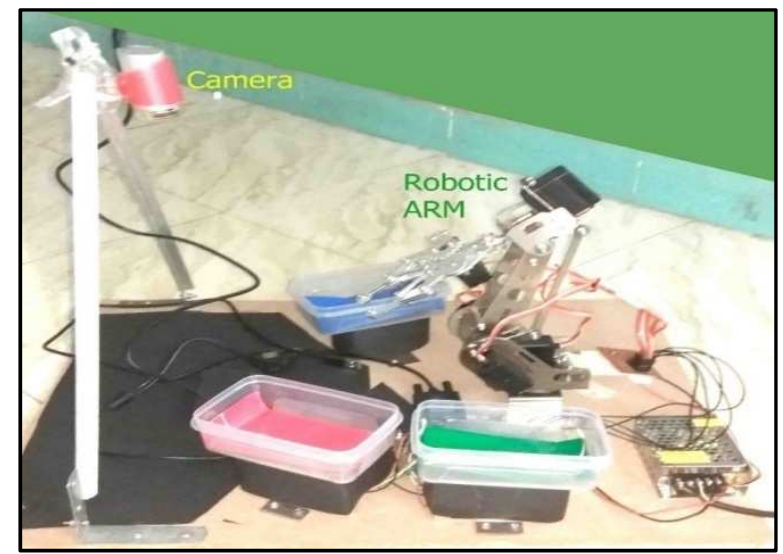

Figure 5: Implemented Object Sorting System

The entire system is powered with $5 \mathrm{~V}$ switched mode power supply which provides supply to microcontroller unit, color sensors, and robotic arm servo motors.

\section{CONCLUSIONS}

The color-based object recognition and intelligently dropping in the respective color bucket is a novel approach development is shown in this paper. The implemented arm can sort the object efficiently and accurately even after a reshuffling of bucket locations. The system is applicable where large volumes of objects are to be sorted based on their color. The system is easy to implement and cost effective which provides better accuracy and efficiency and also possesses rigid mechanical structure hence practically applicable in industrial environments. 


\section{REFERENCES}

1. Information on the HSV color space, cs.haifa.ac.il/hagit/courses/ist/Lectures/Demos/ColorApplet/me/infohsv.html

2. $\quad R G B$ color space https://en.wikipedia.org/wiki/RGB_color_space

3. Amitesha Sachdeva1, Mahesh Gupta2, Manish Pandey3, Prabham Khandelwal4” Development Of Industrial Automatic Multi Colour Sorting and Counting Machine Using Arduino Nano Microcontroller and TCS3200 Colour Sensor" The International Journal of Engineering and Science (IJES) || Volume || 6 || Issue || 4

4. Wikipedia Creative Commons. http://en.wikipedia.org/wiki/file:schematic diagram of the human eye en.svg.

5. Trinesh, T. M., and a. V. Bongale, "Detection and Distinction of Colors Using Color Sorting Robotic Arm in a Pick and Place Mechanism," International Journal of Advanced Research in Engineering, vol. Vol. 4, 2018

6. N. R. Vange, A. V. Nar, D. B. Surve, A. P. Trimukhe, M. M. Patil, and R. A. Patil, "Pic-O-Place Robotic Arm Object Sorting Robotic Arm Based on Color Sensing Mechanism," International Journal of Emerging Technology and Advanced Engineering, vol. 5, May 2015.

7. U. Amin, G. Ahmad, N. Liaqat, M. Ahmed, and S. Zahoor, "Detection \& Distinction of Colors using Color Sorting Robotic Arm in a Pick \& Place Mechanism," Paper ID, vol. 20131882.

8. Sharma, U., \& Kumar, R. A. J. E. S. H. (2014). Modeling of smart capacitive humidity sensor using ANN. International Journal of Research in Engineering \& Technology, 2, 265-272.

9. D. V. K. Reddy, "Sorting of Objects Based on Colour By pick And Place Robotic Arm and with Conveyor Belt Arrangement," 2014.

10. S. Wanve and B. G. Gawalwad, "Automatic color object sorting system," International Journal of modern Trends in Engineering and research, july 2015.

11. Vishnu r. Kale, v. A. Kulkarni “automation of object sorting system using pick \&place robotic arm \& image processing” Proceedings of 3rd IRAJ International Conference, 5th January 2014, Mumbai, India.

12. HSV(Hue, Saturation and Value) www.tech-faq.com/hsv.html

13. Convert from HSV to RGB Color Space https://www.mathworks.com/help/images/convert-from-hsv-to-rgb-colorspace.html 